\title{
Cyclostationary Feature Detection Based Blind Approach for Spectrum Sensing and Classification
}

\author{
Gemi Rachel GEORGE, Samuel Chris PREMA \\ Dept. of Avionics, Indian Institute of Space Science and Technology, Trivandrum, Kerala, India \\ gemirachelg@gmail.com, chrisprema@iist.ac.in \\ Submitted July 30, 2018 / Accepted October 13, 2018
}

\begin{abstract}
A Spectrum Sensing (SS) device, regardless of its location, should be able to detect the presence of signal over noise. In certain applications, SS should be able to correctly identify and classify the received signal. These functions are to be performed with little or no prior information about the incoming signal or channel noise. Cyclostationary Feature Detection (CFD) can be used to detect primary users (PU) using periodicity in autocorrelation of the modulated signals. These algorithms attempt to differentiate signal from noise based on the uncorrelated nature of noise. CFD is often considered as a semi-blind approach, since it requires prior information about the PU signal for detection. For identification and classification of PU signal, existing algorithms make use of CFD and neural networks. This paper proposes a novel algorithm to obtain completely blind detection performance based on CFD. Classification of PU signals is based on the basic statistics regarding cyclic spectrum. Further, an algorithm is formulated to identify modulation scheme of the signal and classify it without making use of any training algorithms. The proposed approach is capable of detecting $P U$ reliably for $S N R$ as low as $-8 d B$ with no prior information about PU or noise in the channel.
\end{abstract}

\section{Keywords}

Spectrum Sensing (SS), Cyclostationary Feature Detection (CFD), Spectral Correlation Density function (SCD)

\section{Introduction}

The advances in wireless communication and emerging wireless multimedia applications have led to a huge demand for radio spectrum. Spectrum scarcity has become one of the major problems hindering the growth of wireless communication. Different solutions like Free Space Optical (FSO) communication and Cognitive Radio (CR) have emerged for efficient utilization of spectrum [1]. CR is a promising solution provided by the concept of dynamic spectrum access, where the users intelligently sense the spectrum and access the vacant bands [2]. It is an intelligent radio and network technology that can detect the available spectrum bands and adjust its transmission parameters accordingly. Moreover in some wireless applications and services there is a requirement to correctly identify and classify the received signal, with little or no prior information about the incoming signal or channel noise. Different techniques for SS include energy detection, matched filtering, covariance and Eigen value based approaches [3], [4]. Cyclostationary Feature Detection (CFD) makes use of the periodicity in auto-correlation of the modulated signals. Exploiting the uncorrelated nature of the noise, the above algorithms attempt to differentiate signal from noise [5], [6]. The computed cyclic autocorrelation function can also be used to detect the presence of OFDM signals [7], [8]. In [9], a generalized likelihood ratio test for detecting the presence of cyclostationarity using multiple cyclic frequencies is proposed. The detection has been expanded to multiple SUs by making use of cooperative sensing, but assumes the cyclic frequencies are known apriori. Signal detection and preprocessing for signal classification using cycle frequency domain profile is discussed in [10]. A FPGA implementation of a CFD using decimation to control detection time and improve the probability of detection is discussed in [11], assuming test statistic to be $\chi_{2}^{2}$ distributed and hypothesis tests are performed with known cyclic frequencies. Frequency Shift (FRESH) filters are used in [12] to enable SS at low SNR by estimating a cyclostationary signal using its spectral coherence properties. In [13], a performance study of the cyclostationarity based digital modulation classification schemes are discussed. Different classifiers like artificial neural networks, support vector machine, Naive Bayes etc. were compared for performances. CFD, using a crest factor developed from the cyclic spectrum and a threshold formed using the noise information that is obtained in the absence of PU, is also applied for SS [14].

Existing techniques make use of the PU information for detection and hence CFD is often considered as a semi-blind approach. An attempt on blind spectrum sensing using cyclostationary features, with decision criteria based on practical assumptions, is detailed in [15]. Most of the classification algorithms use Neural networks to achieve correct identification. This work is an attempt to obtain completely blind detection performance based on CFD. Further, an algorithm 
has been formulated to identify the modulation scheme of the signal received without making use of Neural networks. Simulation results are shown to validate the effectiveness of the proposed algorithms. Section 2 and 3 explain the CFD and the SCD function. Section 4 explains the proposed algorithm for detection and classification, while Section 5 and 6 illustrates the results and provides a conclusion.

\section{Cyclostationary Features}

A signal having statistical properties that vary cyclically with time is known as a cyclostationary process, and can be viewed as multiple interleaved stationary processes. The underlying concept for cyclostationarity is that sine waves can be generated from random data, by applying certain nonlinear transformations. Thus, a continuous signal is said to be cyclostationary of order $n$, if and only if there exists some $n^{\text {th }}$ order non-linear transformation that will generate finite amplitude additive sine wave components [5]. Therefore, a continuous signal $x(t)$ contains a finite amplitude additive sine wave component with frequency $\alpha, \alpha \neq 0$, if the Fourier coefficient $M_{x}^{\alpha}$ is non zero.

$$
M_{x}^{\alpha}=\lim _{T \rightarrow \infty} \frac{1}{T} \int_{-T / 2}^{T / 2} x(t) \mathrm{e}^{-\mathrm{j} 2 \pi \alpha t} \mathrm{~d} t .
$$

For second order cyclostationarity, the non linear transformation required for the signal is:

$$
y_{\tau}(t)=x(t+\tau / 2) x^{*}(t-\tau / 2)
$$

where * stands for complex conjugation. Thus, it can be concluded that a process is second order cyclostationary, if its mean and time varying autocorrelation function are periodic in time. i.e.,

$$
\begin{gathered}
R_{x}(t, \tau)=\mathbb{E}\left\{x(t+\tau / 2) x^{*}(t-\tau / 2)\right\}, \\
R_{x}(t+T, \tau)=R_{x}(t, \tau)
\end{gathered}
$$

where $T$ is the hidden cyclic period. The Fourier coefficients of $R_{x}(t, \tau)$ is called the Cyclic Autocorrelation Function (CAF) [5]. For any second order cyclostationary process, the CAF is non zero only for a set of cyclic frequencies $\alpha \neq 0$. In communication signals, cyclostationarity is caused by modulation or coding, and at times, it is even intentionally produced to aid channel estimation and synchronization. These signals have cyclic frequencies that are related to its carrier frequency, symbol rate and its harmonics, guard period, channel coding scheme etc. On the other hand, noise is wide sense stationary and exhibits no correlation. This basic difference between signal and noise can be used to detect the presence of a PU in a spectrum band. Even though, the method is computationally more complex, it can be modified to work with no prior information about the PU signal or the noise information in the channel. Moreover, it can be used to identify the modulation scheme of the incoming signal and can be extended to predict the carrier frequency used for the modulation.

\section{SCD Function}

A process $x(t)$ is second order cyclostationary, if its mean and time varying autocorrelation function are periodic in time. This transformation can be used to bring out the cyclic frequencies hidden in the signal. The autocorrelation function is given as [5]:

$$
R_{x}(t, \tau)=E\left\{x(t+\tau / 2) x^{*}(t-\tau / 2)\right\} .
$$

Since it is periodic, it can be decomposed into its Fourier series as:

$$
R_{x}(t, \tau)=\sum_{k=1}^{M} R_{x}^{k \alpha_{0}}(\tau) \mathrm{e}^{\mathrm{j} 2 \pi k \alpha_{0} t} .
$$

The fundamental cyclic frequency is $\alpha_{\mathrm{o}}=1 / T_{\mathrm{o}}$, where $T_{\mathrm{o}}$ is the hidden period and $M$ represents the rank of the last harmonic.

The Fourier coefficients of $R_{x}(t, \tau)$ are called Cyclic Autocorrelation Function (CAF).

$$
R_{x}^{k \alpha_{\mathrm{o}}}(\tau)=\lim _{T \rightarrow \infty} \frac{1}{T} \int_{-T / 2}^{+T / 2} R_{x}(t, \tau) \mathrm{e}^{-\mathrm{j} 2 \pi k \alpha_{\mathrm{o}} t} \mathrm{~d} t
$$

where $T$ is the time duration used to evaluate the CAF. The Fourier transform of the CAF is called the Spectral Correlation Density function (SCD).

$$
S_{x}^{\alpha}(f)=\int_{-\infty}^{+\infty} R_{x}^{\alpha}(\tau) \mathrm{e}^{-\mathrm{j} 2 \pi f \tau} \mathrm{d} \tau .
$$

As given in [14], equation (7) can be approximated as:

$$
\begin{gathered}
R_{x}^{\alpha}(\tau)=\lim _{T \rightarrow \infty} \frac{1}{T} \int_{-T / 2}^{+T / 2} x(t+\tau / 2) x^{*}(t-\tau / 2) \mathrm{e}^{-\mathrm{j} 2 \pi \alpha t} \mathrm{~d} t \\
R_{x}^{\alpha}(\tau)=\lim _{T \rightarrow \infty} \frac{1}{T} \int_{-T / 2}^{+T / 2} x(t+\tau / 2) \mathrm{e}^{-\mathrm{j} 2 \pi \frac{\alpha}{2}(t+\tau / 2)} \\
x^{*}(t-\tau / 2) \mathrm{e}^{-2 j \pi \frac{\alpha}{2}(t-\tau / 2)} \mathrm{d} t
\end{gathered}
$$

Denoting $y(t)=x^{*}(t-\tau / 2) \mathrm{e}^{-2 \mathrm{j} \pi \frac{\alpha}{2}(t-\tau / 2)}$. Thus the SCD is expressed as:

$$
\begin{gathered}
S_{x}^{\alpha}(f)=\mathbb{F} \mathbb{T}\left\{R_{x}^{\alpha}(\tau)\right\}, \\
S_{x}^{\alpha}(f)=\lim _{T \rightarrow \infty} \frac{1}{T} \mathbb{F} \mathbb{T}\left\{y(\tau) * y^{*}(-\tau)\right\}, \\
S_{x}^{\alpha}(f)=\lim _{T \rightarrow \infty}\left\{\frac{1}{T} X_{T}(f+\alpha / 2) X_{T}^{*}(f-\alpha / 2)\right\} .
\end{gathered}
$$

In equation (12) $X_{T}(f)$ is the Fourier transform of the product of $x(t)$ and a rectangular window of width $T$.

$$
X_{T}(f)=\int_{-T / 2}^{T / 2} x(t) \mathrm{e}^{-\mathrm{j} 2 \pi f t} \mathrm{~d} t .
$$




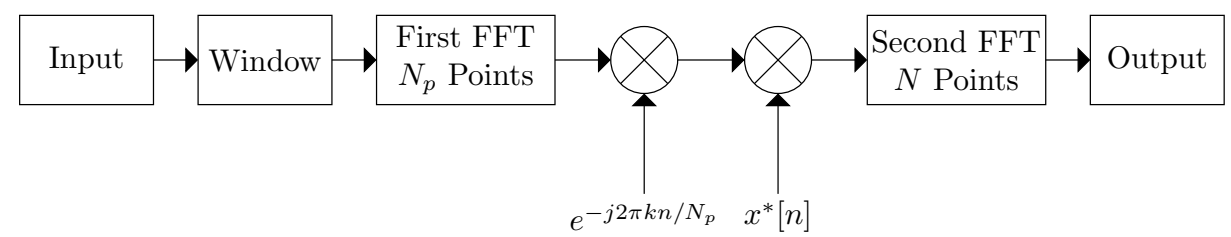

Fig. 1. SSCA block diagram.

Spectral correlation density function can be estimated using algorithms that either average in frequency or in time. Time smoothing algorithms are often considered to be computationally better for general cyclic spectral analysis. The most common time smoothing algorithms are FFT Accumulation Method (FAM) and Strip Spectral Correlation Algorithm (SSCA) [16]. In [17] the estimation procedure under different conditions of sampling frequency and signal frequency are studied to conclude that any inaccuracy in frequency has only acceptable consequences.

\subsection{Strip Spectral Correlation Algorithm}

Strip Spectral Correlation Algorithm (SSCA) is one of the most common time smoothing algorithms used to estimate the spectral correlation density function. The present study has made use of SSCA to compute the SCD function of the received signal samples.

Given an observation time of $\Delta t$, an estimation of the SCD can be obtained as a time smoothed cyclic periodogram,

$$
S_{x}^{\alpha}(f) \approx S_{x_{T_{\mathrm{w}}}}^{\alpha}(t, f)_{\Delta t}=\frac{1}{\Delta t} \int_{t-\Delta t / 2}^{t+\Delta t / 2} S_{x_{T_{x}}}(u, f) \mathrm{d} u
$$

where

$$
S_{x_{T_{\mathrm{w}}}}(u, f)=\frac{1}{T_{\mathrm{w}}} X_{T_{\mathrm{w}}}(u, f+\alpha / 2) X_{T_{\mathrm{w}}}^{*}(u, f-\alpha / 2) .
$$

Here $X_{T_{\mathrm{w}}}$ is the short time Fourier Transform and $T_{\mathrm{w}}$ is its window width.

The basic operations involved in estimating the SCD using SSCA is shown as a block diagram in Fig. 1 [16]. The steps in SSCA are as follows:

1. The input sample of length $N$ is divided into $P$ blocks of $N_{\mathrm{p}}$ samples each. $L$ data samples are skipped between two successive blocks of $N_{\mathrm{p}}$ samples. The values of $L, P$ and $N_{\mathrm{p}}$ are fixed according to the resolution in frequency and cyclic frequency.

$$
\begin{gathered}
N_{\mathrm{p}}=\frac{F_{\mathrm{s}}}{\Delta f}, \\
L=\frac{N_{\mathrm{p}}}{4}, \\
P=\frac{F_{\mathrm{s}}}{L \Delta \alpha} .
\end{gathered}
$$

2. A Hamming window $w(n)$ is applied for each block.
3. $N_{\mathrm{p}}$ points FFT is computed for each block to obtain the complex envelope $X_{T_{\mathrm{w}}}(n, f)$. It is then downshifted in frequency to obtain $X_{T_{\mathrm{w}}}(n, f+\alpha / 2)$.

4. The complex demodulated sequence is directly multiplied by the complex conjugate of the signal.

5. The resulting signal is then time smoothed by means of an $N$-point FFT $(N=P * L)$.

Thus we have an estimate of the SCD function of the given signal. The spectral density functions for different modulation schemes and theoretical proof for the existence of peaks at different frequencies are detailed in [18].

\subsection{Examples}

For the present study, the modulation schemes under consideration are: FM, AM, BPSK, QPSK, BFSK, QAM and PAM. As a first step, signals were generated for each of these modulation schemes and the SCD is obtained for each of them under various modulation parameters. A few sample graphs showing the SCD plotted against frequency and cyclic frequency are given in Fig. 2.

\section{Proposed Algorithm}

Most of the existing approaches for SS based on CFD uses primary user information and noise information for detection. Even the latest works on cyclostationary feature detection require noise features to compute the threshold for hypothesis testing [14]. Classification for the modulation schemes is attempted using artificial neural networks and other such similar approaches. The present study makes an attempt to devise a novel approach for detection and classification of PU signals that uses the basic statistics regarding the cyclic spectrum. The following information are obtained from the SCD for detection:

1. Peak value of the SCD.

2. Number of occurrences of the peak.

3. Distribution of the SCD values.

4. The spread of the peaks in frequency and cyclic frequency.

From this information, parameters are formulated accordingly for detection and classification. Monte Carlo simulations were carried out to study the value of the formulated parameters under different conditions. 


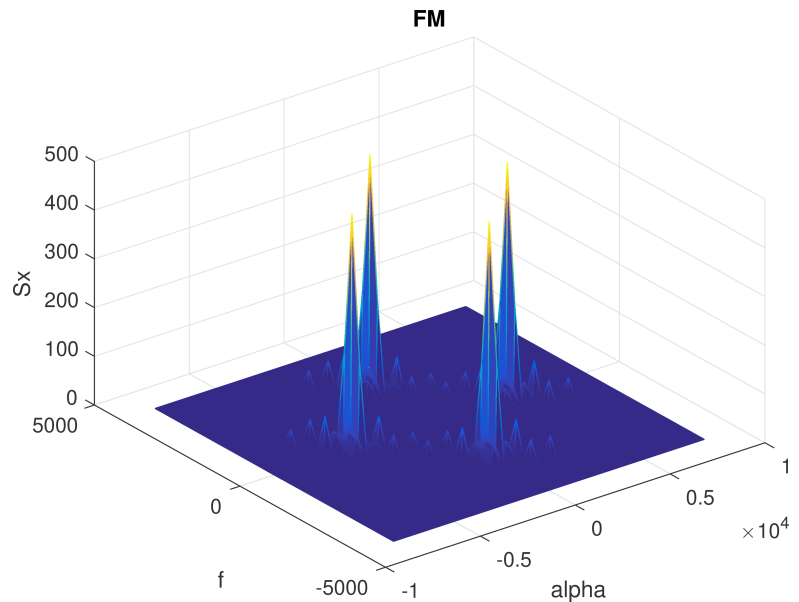

(a) Frequency Modulation.

FM

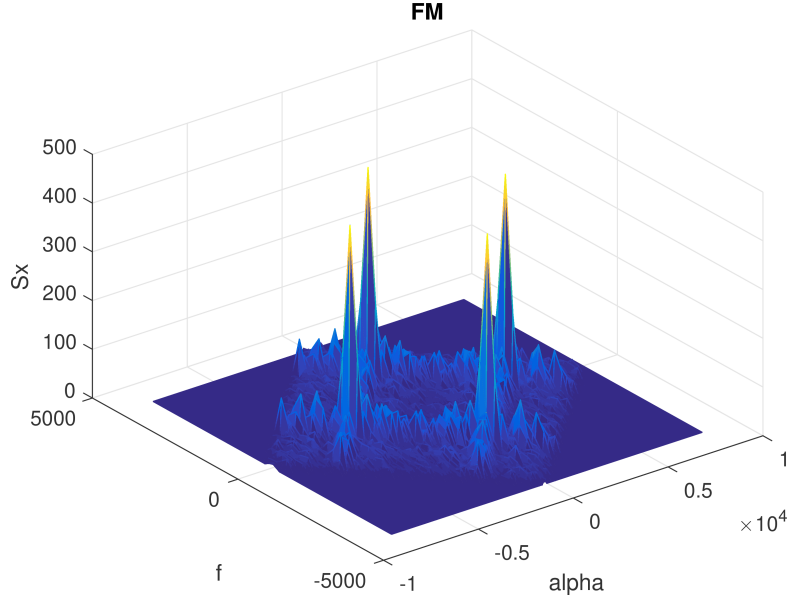

(c) FM with $0 \mathrm{~dB}$ noise.

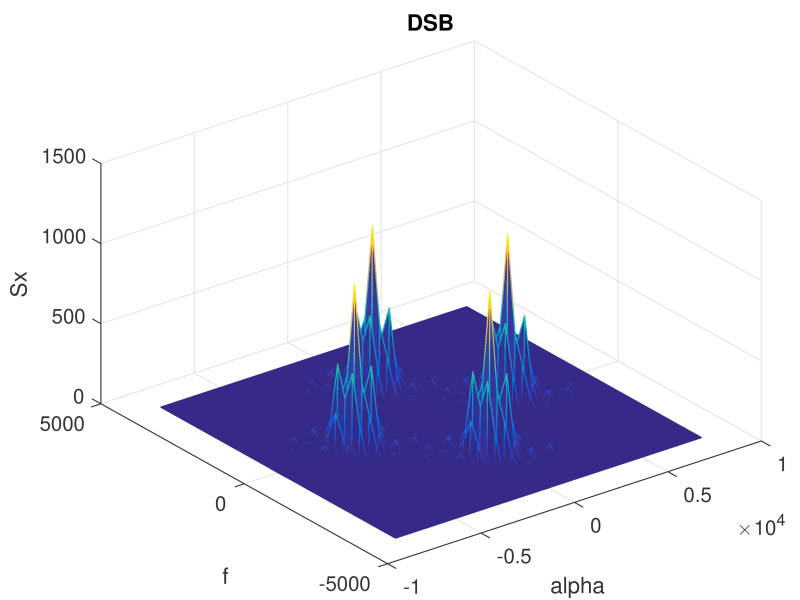

(b) Amplitude Modulation (DSB).

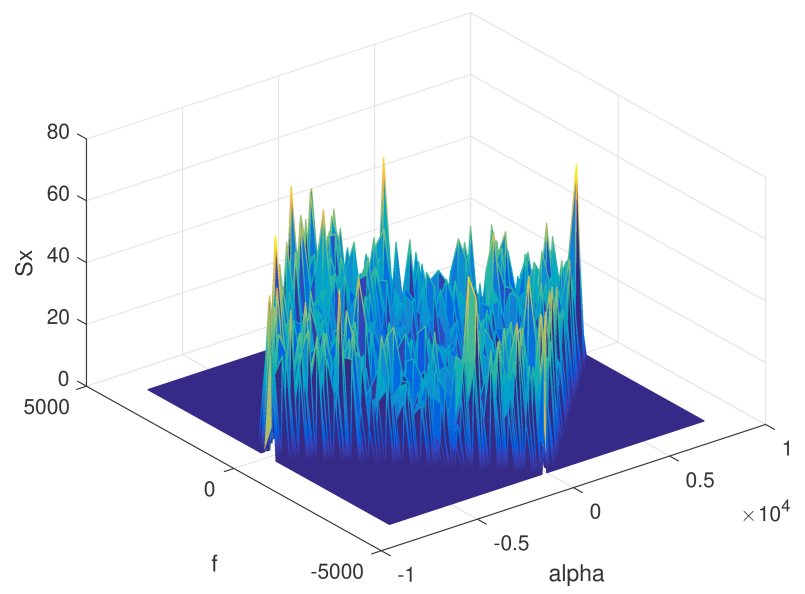

(d) AWGN noise $(\mathrm{dBw})$.

Fig. 2. Sample SCD.

\subsection{Detection}

From the different simulations carried out for PU signals and noise, it was observed that all of the signals have spectral peaks along $f=0$ or $\alpha=0$. But this is not the case with noise, where the maximum is anywhere along the distribution. This basic difference can be used to detect signals from noise. Thus a test statistic is formulated as:

$$
T=\frac{\operatorname{Peak}(f, \alpha) \times f_{\mathrm{len}} \times a_{\mathrm{len}}}{P \times f_{\text {num }} \times a_{\text {num }}}
$$

where

Peak $(f, \alpha)$ : Number of dominant peaks at $f=0$ or $\alpha=0$,

$P$ : Total number of peaks in the SCD,

$f_{\text {len }}$ : Total number of frequencies considered,

$a_{\text {len }}$ : Total number of cyclic frequencies considered,

$f_{\text {num }}$ : Total number of frequencies occupied by significant SCD values,

$a_{\text {num }}$ : Total number of cyclic frequencies occupied by significant SCD values.
As observed in Fig. 2d, the SCD values for noise is spread across the frequencies and cyclic frequencies. This brings $f_{\text {num }} \approx f_{\text {len }}$ and $a_{\text {num }} \approx a_{\text {len }}$. Further, noise SCD does not necessarily have peaks at $f=0$ or $\alpha=0$. This reduces the value of the statistic close to zero. In effect, the value of the statistic is very small when the received input contains just noise. On the other hand, if a signal is present, the statistic assumes significant values. This forms the basis for detection.

\subsection{Classification}

Using a similar approach, an attempt is made to classify the signals based on the modulation scheme. Multi-level checks were devised for the classification based on the data obtained. The different checks used for the classification are formulated based on the following parameters:

1. Number of peaks in SCD.

2. Using the statistic:

$$
d=\frac{l_{\alpha}}{l_{f}}
$$


where, $l_{\alpha}$ is the number of cyclic frequencies for which the SCD value is very high and $l_{f}$ is the number of frequencies for which the SCD value is very high.

3. Number of peaks of the SCD at $f=0$.

These parameters have different values for each modulation scheme and thus can be used to identify the signal present in the received input.
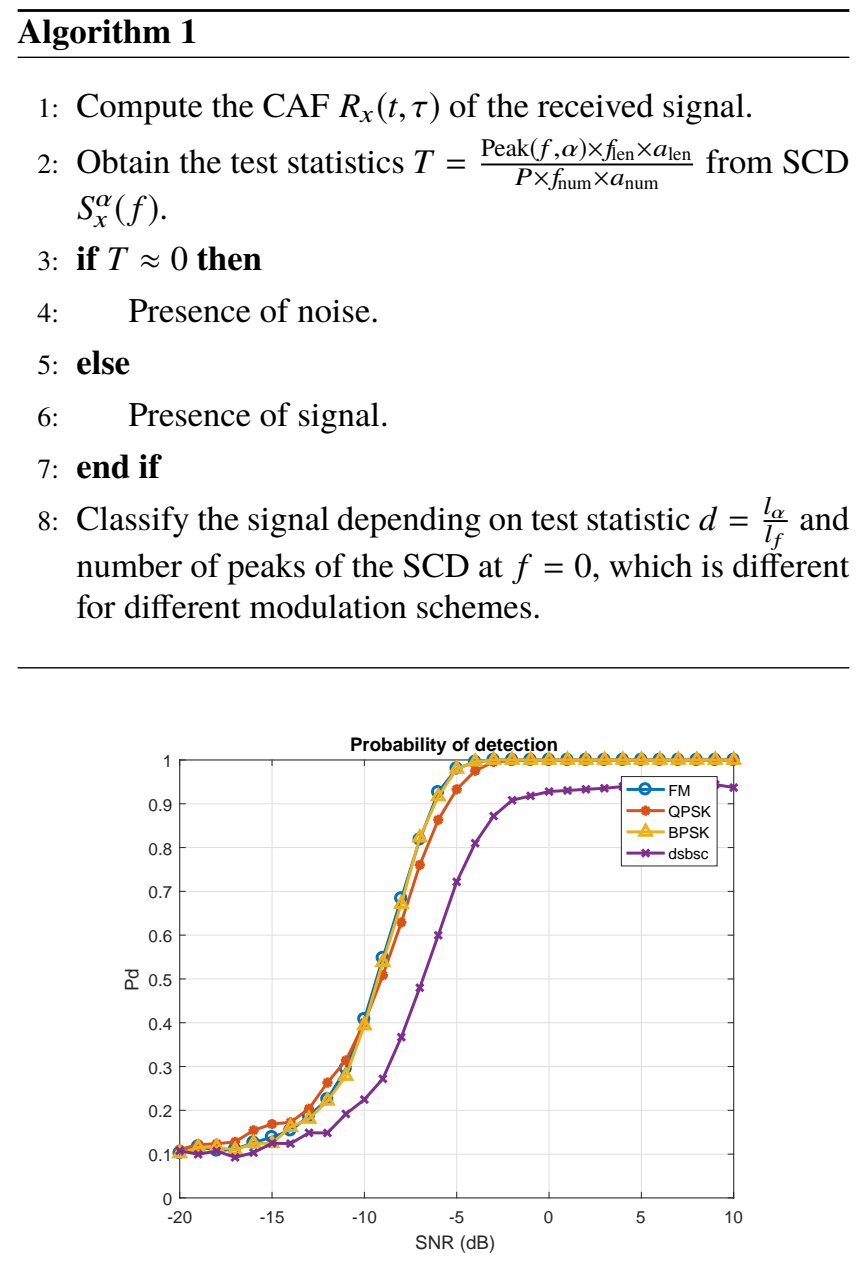

Fig. 3. Detection probability at different SNR.

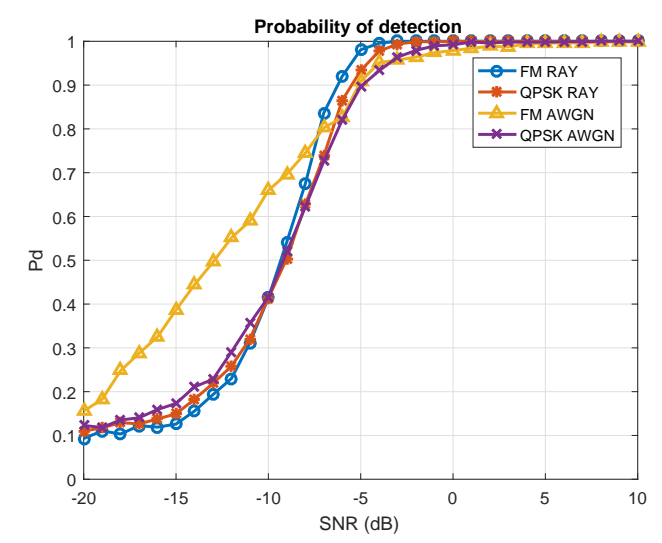

Fig. 5. Detection performance comparison in AWGN and Rayleigh channels.

\section{Results and Discussion}

In order to understand the performance of the algorithms, the probability of detection was calculated for different modulation schemes with varying SNR. Figure 3 shows the detection performance for AM, FM, QPSK and BPSK modulation schemes for different SNR values.

The algorithm is able to detect both analog modulated as well as digital modulated signals. The technique used does not depend on a fixed probability of false alarm and hence the $P_{\mathrm{fa}}$ was simulated at different SNR to gain a better understanding into its performance and shown in Fig. 4.

In order to analyse the performance of the algorithm under realistic channel conditions, AWGN and Rayleigh channel were simulated for detection of FM and QPSK modulation. Figure 5 shows the detection performance of the proposed algorithm for varying SNRs in both the channel conditions. The proposed algorithm is also compared with an existing non blind scheme [10] of detection to validate its significance. Figure 6 shows that the proposed blind scheme is comparable in performance to that of the existing non blind approach. In order to evaluate the performance of the classification algorithms, the probability of correct classification was obtained at different values of SNR. Figure 7 shows a sample result for the classification of FM, AM and QPSK signals.

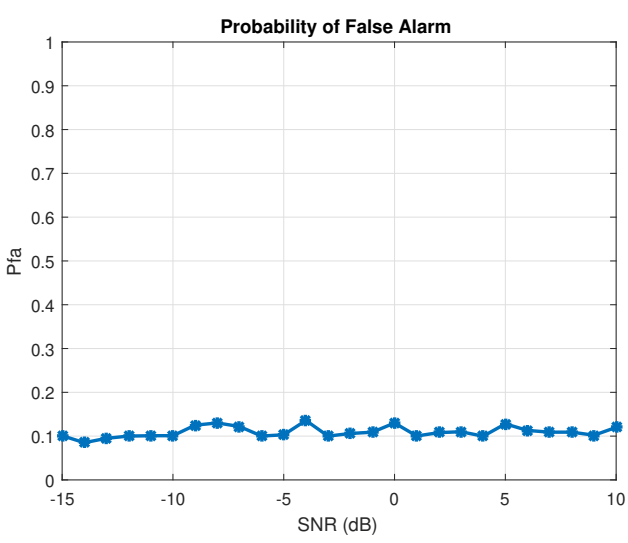

Fig. 4. Probability of False Alarm at different SNR.

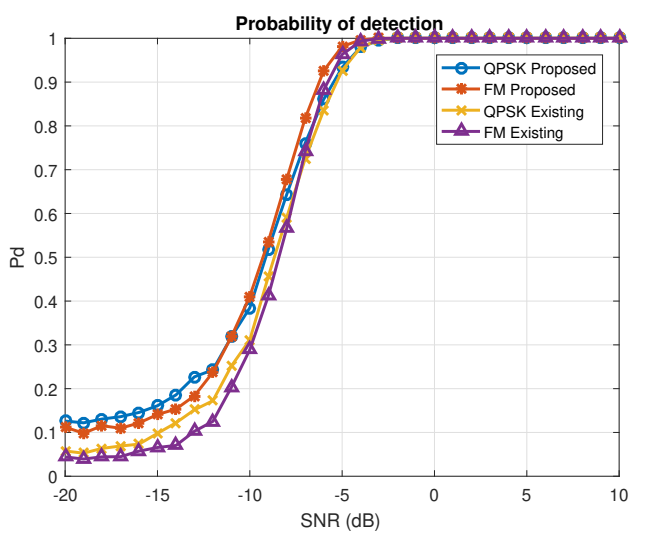

Fig. 6. Comparison with existing methods for varying SNR. 


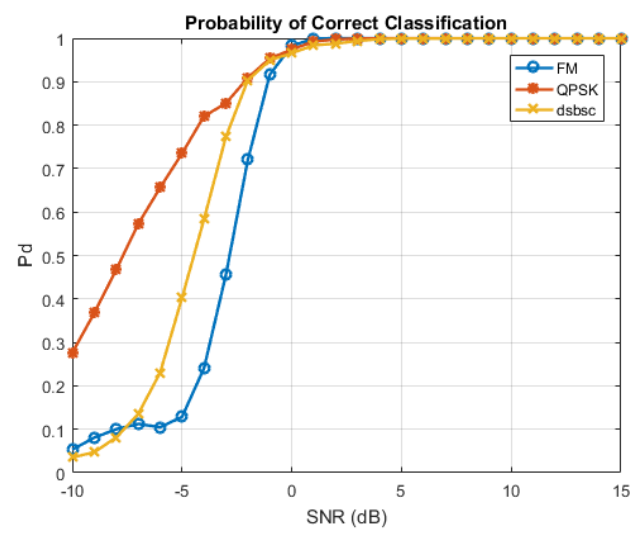

Fig. 7. Probability of correct classification at different SNR.

\section{Conclusion}

The proposed blind approach is able to detect the presence of the PU reliably for SNR as low as $-8 \mathrm{~dB}$ with no prior information about the $\mathrm{PU}$ or the noise in the channel. The algorithm functions with a probability of false alarm of approximately 0.1 for all values of SNR. This remains almost constant throughout the range of SNR values. It was also observed that with no prior information about the signal, the algorithm is able to achieve good classification probability for SNR as low as $-3 \mathrm{~dB}$ and performs with $100 \%$ accuracy above $0 \mathrm{~dB}$. This approach provides a means to classify the received signal based on its modulation scheme with no prior information about the primary users in the network.

\section{References}

[1] KHAN, M. N., GILANI, S. O., JAMIL, M., et al. Maximizing throughput of hybrid FSO-RF communication system: An algorithm. IEEE Access, 2018, vol. 6, p. 30039-30048. DOI: 10.1109/Access.2018.2840535

[2] HAYKIN, S. Cognitive radio: brain-empowered wireless communications. IEEE Journal on Selected Areas in Communications, 2005, vol. 23, no. 2, p. 201-220. DOI: 10.1109/JSAC.2004.839380

[3] ZENG, Y., LIANG, Y.-C. Eigenvalue-based spectrum sensing algorithms for cognitive radio. IEEE Transactions on Communications, 2009 , vol. 57 , no. 6, p. 1784-1793. DOI: 10.1109/TCOMM.2009.06.070402,

[4] ZENG, Y., LIANG, Y.-C. Spectrum-sensing algorithms for cognitive radio based on statistical covariances. IEEE Transactions on Vehicular Technology, 2009, vol. 58, no. 4, p. 1804-1815. DOI: $10.1109 /$ TVT.2008.2005267

[5] GARDNER, W. A. Cyclostationarity in Communications and Signal Processing. Piscataway, NJ: IEEE Press, sponsored by IEEE Communications Society, 1994. ISBN: 0780310233

[6] ZHANG, J., ZHANG, L., HUANG, H., JING, X. J. Improved cyclostationary feature detection based on correlation between the signal and noise. In Proceedings of the International Symposium on Communications and Information Technologies (ISCIT). Qingdao (China), 2016, p. 611-614. DOI: 10.1109/ISCIT.2016.7751705

[7] SEBESTA, V., MARSALEK, R., FEDRA, Z. OFDM signal detector based on cyclic autocorrelation function and its properties. Radioengineering, 2011, vol. 20, no. 4, p. 926-931.
[8] PREMA, G., GAYATRI, P. Blind spectrum sensing method for OFDM signal detection in cognitive radio communications. In Proceedings of the International Conference on Communication and Network Technologies (ICCNT). Sivakasi (India), 2014, p. 42-47. DOI: $10.1109 /$ CNT.2014.7062722

[9] LUNDEN, J., KOIVUNEN, V., HUTTUNEN, A., POOR, H. V. Spectrum sensing in cognitive radios based on multiple cyclic frequencies. In Proceedings of the International Conference on Cognitive Radio Oriented Wireless Networks and Communications. Orlando (USA), 2007, p. 37-43. DOI: 10.1109/CROWNCOM.2007.4549769

[10] KIM, K., AKBAR, I. A., BAE, K. K., et al. Cyclostationary approaches to signal detection and classification in cognitive radio. In Proceedings of the IEEE International Symposium on New Frontiers in Dynamic Spectrum Access Networks (DySPAN). Dublin (Ireland), 2007, p. 212-215. DOI: 10.1109/DYSPAN.2007.35

[11] TURUNEN, V., KOSUNEN, M., HUTTUNEN, A., et al. Implementation of cyclostationary feature detector for cognitive radios. In Proceedings of the International Conference on Cognitive Radio Oriented Wireless Networks and Communications (CROWNCOM). Hannover (Germany), 2009, p. 1-4. DOI: 10.1109/ISSPIT.2016.7886025

[12] SAGGAR, H., MEHRA, D. Cyclostationary spectrum sensing in cognitive radios using fresh filters. arXiv:1312.5257, 2013, $6 \mathrm{p}$.

[13] SATIJA, U., MANIKANDAN, M., RAMKUMAR, B. Performance study of cyclostationary based digital modulation classification schemes. In Proceedings of the International Conference on Industrial and Information Systems (ICIIS). Gwalior (India), 2014, p. 1-5. DOI: 10.1109/ICIINFS.2014.7036609

[14] KADJO, J.-M., YAO, K. C., MANSOUR, A. Blind detection of cyclostationary features in the context of cognitive radio. In Proceedings of the International Symposium on Signal Processing and Information Technology (ISSPIT). Limassol (Cyprus), 2016, p. 150-155. DOI: 10.1109/ISSPIT.2016.7886025

[15] GATO, L. M., MARTÍNEZ, L., TORRES, J. Blind spectrum sensing based on cyclostationary feature detection. In Proceedings of the Iberoamerican Congress on Pattern Recognition. Montevideo (Uruguay), 2015, p. 535-542. DOI: 10.1007/978-3-319-25751-8/64

[16] ROBERTS, R. S., BROWN, W. A., LOOMIS, H. H. Computationally efficient algorithms for cyclic spectral analysis. IEEE Signal Processing Magazine, 1991, vol. 8, no. 2, p. 38-49. DOI: 10.1109/79.81008

[17] SEBESTA, V. Estimating a spectral correlation function under the conditions of imperfect relation between signal frequencies and a sampling frequency. Radioengineering, 2010, vol. 19, no. 1, p. 1-5.

[18] DA COSTA, E. L. Detection and Identification of Cyclostationary Signals. Ph.D. thesis, 1996. Naval Postgraduate School, Monterey (USA)

\section{About the Authors}

Gemi Rachel GEORGE was born in 1996. She received her Bachelors Degree in Avionics from Indian Institute of Space Science and Technology. Her research interests include communication systems and control systems.

Samuel Chris PREMA was born in 1979. She received her Bachelors Degree in Electronics and Communication, Masters Degree in Applied Electronics and Ph.D. in Avionics Department from Indian Institute of Space Science and Technology. Her research interests include communication and multirate filter banks for wideband spectrum sensing in cognitive radios. She is currently a faculty in Avionics Department, Indian Institute of Space Science and Technology. 